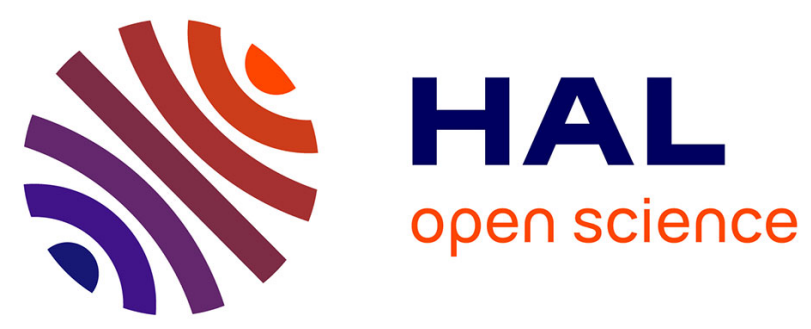

\title{
Approche chronologique de quelques sites ornés paléolithiques du Périgord par datation Carbone 14, en spectrométrie de masse par accélérateur, de leur mobilier archéologique
}

Norbert Aujoulat, Jean-Jacques Cleyet-Merle, Jean Gaussen, Nadine Tisnerat, Hélène Valladas

\section{To cite this version:}

Norbert Aujoulat, Jean-Jacques Cleyet-Merle, Jean Gaussen, Nadine Tisnerat, Hélène Valladas. Approche chronologique de quelques sites ornés paléolithiques du Périgord par datation Carbone 14, en spectrométrie de masse par accélérateur, de leur mobilier archéologique. PALEO : Revue d'Archéologie Préhistorique, 1998, 10 (1), pp.319-323. 10.3406/pal.1998.1144 . hal-02497244

\author{
HAL Id: hal-02497244 \\ https://hal.science/hal-02497244
}

Submitted on 8 Feb 2021

HAL is a multi-disciplinary open access archive for the deposit and dissemination of scientific research documents, whether they are published or not. The documents may come from teaching and research institutions in France or abroad, or from public or private research centers.
L'archive ouverte pluridisciplinaire HAL, est destinée au dépôt et à la diffusion de documents scientifiques de niveau recherche, publiés ou non, émanant des établissements d'enseignement et de recherche français ou étrangers, des laboratoires publics ou privés. 
Approche chronologique de quelques sites ornés paléolithiques du Périgord par datation Carbone 14, en spectrométrie de masse par accélérateur, de leur mobilier archéologique/Chronological approach to a few decorated Paleolithic sites in the Perigord based on C14 dating of associated archaeological objects Jean-Jacques Cleyet-Merle, Jean Gaussen, Nadine Tisnerat, Hélène Valladas

\begin{abstract}
Chronological approach to a few decorated Paleolithic sites in the Perigord based on C14 dating of associated archaeological objects. Several radiocarbon dates of archaeological sites with parietal art in Périgord (Gabillou, Jamblancs, Lascaux) recently obtained, ranging from 17180 to $18600 \mathrm{BP}$, strengthen their chronological attribution to the Late Solutrean and the Early Magdalénien.
\end{abstract}

\title{
Résumé
}

Plusieurs datations radiocarbone du contexte archéologique de trois sites d'art pariétal périgourdins (Gabillou, Jamblancs, Lascaux,) récemment obtenues, échelonnées entre 17180 et $18600 \mathrm{BP}$, confortent leur attribution chronologique à la période couvrant la fin du Solutréen et le début du Magdalénien.

\section{Citer ce document / Cite this document :}

Cleyet-Merle Jean-Jacques, Gaussen Jean, Tisnerat Nadine, Valladas Hélène. Approche chronologique de quelques sites ornés paléolithiques du Périgord par datation Carbone 14, en spectrométrie de masse par accélérateur, de leur mobilier archéologique/Chronological approach to a few decorated Paleolithic sites in the Perigord based on $\mathrm{C} 14$ dating of associated archaeological objects. In: Paléo. N. 10,1998. pp. 319-323;

doi : https://doi.org/10.3406/pal.1998.1144

https://www.persee.fr/doc/pal_1145-3370_1998_num_10_1_1144

Fichier pdf généré le 24/05/2018 


\title{
APPROCHE CHRONOLOGIQUE DE QUELQUES SITES ORNÉS PALÉOLITHIQUES DU PÉRIGORD PAR DATATION CARBONE 14 EN SPECTROMÉTRIE DE MASSE PAR ACCÉLÉRATEUR DE LEUR MOBILIER ARCHÉOLOGIQUE
}

\author{
N. AUJOULAT ${ }^{(1)}$, J.-J. CLEYET-MERLE ${ }^{(2)}, J^{\text {J. GAUSSEN }}{ }^{(3)}$, \\ N. TISNERAT ${ }^{(4)}, H$. VALLADAS ${ }^{(4)}$
}

Résumé : Plusieurs datations radiocarbone du contexte archéologique de trois sites d'art pariétal périgourdins (Gabillou, Jamblancs, Lascaux,) récemment obtenues, échelonnées entre 17180 et $18600 \mathrm{BP}$, confortent leur attribution chronologique à la période couvrant la fin du Solutréen et le début du Magdalénien.

Mots-clés: Datations, Gabillou, Jamblancs, Lascaux, Solutréen, Magdalénien.

Abstract : Chronological approach to a few decorated Paleolithic sites in the Perigord based on C14 dating of associated archaeological objects. Several radiocarbon dates of archaeological sites with parietal art in Périgord (Gabillou, Jamblancs, Lascaux) recently obtained, ranging from 17180 to $18600 \mathrm{BP}$, strengthen their chronological attribution to the Late Solutrean and the Early Magdalenien.

Key-words: Dates, Gabillou, Jamblancs, Lascaux, Solutrean, Magdalenian.

Au cours de cette décennie, les recherches sur l'art pariétal paléolithique ont bénéficié de l'évolution notable des technologies relatives aux méthodes radionucléaires de datation. Ainsi celle du Carbone 14 en spectrométrie de masse par accélérateur - SMA - (Valladas et al. 1992, Clottes et al. 1992, Clottes et al. 1995) permet la datation directe des peintures ou des dessins pariétaux, à partir de prélèvements sur paroi, la quantité minime de matière nécessaire assurant la préservation de l'intégrité du sujet analysé. Des travaux récents, menés aussi bien en Espagne qu'en France, ont contribué, pour certains sites -Niaux, Altamira ou Cosquer- à préciser, voire confirmer, l'attribution chronologique donnée par le contexte archéologique ou l'analyse stylistique comparative. Pour d'autres, on a dû reconsidérer l'estimation faite à partir des méthodes traditionnelles comme à Chauvet en particulier.

Malgré cette avancée remarquable, la totalité de l'iconographie paléolithique ne peut prétendre à un tel traitement, car nombreux sont les sanctuaires où l'étude des matériaux colorants a révélé l'absence de produits charbonneux, l'oxyde de manganèse s'y substituant ; il en est ainsi pour la plupart des grottes de Dordogne, en parti-

1. Centre national de Préhistoire, 38, rue du 26ème R.I., 24000 Périgueux et UMR-CNRS 5808

2. Musée national de Préhistoire, 24620 Les Eyzies de Tayac et UMR-CNRS 5808

3. Rue de la Providence, 24190 Neuvic-sur-l'lsle

4. Laboratoire des Sciences du Climat et de l'Environnement, Unité mixte de recherche CEA-CNRS, Avenue de la Terrasse, 91198 Gif-sur-Yvette Cedex 
culier Lascaux. En outre, le dessin et la peinture sont des formes d'expression moyennement répandues en Périgord, la gravure dominant ce fonds graphique, comme à Combarelles, Bara Bahau ou Gabillou. Ce constat implique que l'on se réfère toujours, et sous certaines conditions, aux données archéologiques issues des fouilles menées à proximité des secteurs ornés.

La récente obtention de datations radiocarbone directes sur les objets issus du contexte archéologique de trois sites périgourdins ayant livré des témoignages artistiques (Jamblancs, Gabillou, Lascaux) justifie la rédaction de cette note d'information.

En 1989, l'un de nous, (J.-J. Cleyet-Merle) avait en effet découvert lors de ses fouilles aux Jamblancs une sagaie à rainure dorsale, en bois de renne (fig. 2), de dimension exceptionnelle $(L=328 \mathrm{~mm}$ ) pratiquement complète, ainsi que plusieurs fragments d'un exemplaire analogue dans un niveau attribuable au Magdalénien moyen/récent. Ces pièces avaient été sommairement publiées dans une note générale (Cleyet-Merle, 1989) dans l'attente de l'achèvement des fouilles et de la recherche de termes de comparaison. Deux datations avaient alors été faites par la méthode classique du Carbone 14 sur quelques fragments d'esquilles osseuses provenant du même niveau archéologique. Les deux dates obtenues, $13790 \pm 120 \mathrm{BP}$ (Gif 8666) et $13900 \pm 110$ BP (Gif 8669), confirmaient donc l'attribution chronologique de cette couche fondée sur l'étude de son matériel lithique. La mise au jour de Magdalénien relativement récent aux Jamblancs était totalement inattendue sur ce site davantage connu pour sa séquence Solutréen supérieur/Magdalénien ancien. Elle devait désormais être prise en compte pour dater des éléments d'art pariétal découverts ${ }^{(1)}$ sous la forme de deux blocs ornés (Peyrony D., 1912 ; Peyrony D. et E., 1934) dans les déblais des anciennes fouilles. S'ils avaient été, (par nécessité logique), datés du Magdalénien ancien, leur relative similitude avec ceux de Laugerie-Haute exhumés dans le Magdalénien III de l'époque n'était pas sans susciter d'éventuels rapprochements. A ce faisceau de présomptions chronologiques s'ajoutait un dernier argument : la découverte aux Jamblancs d'un mammouth gravé sur une écaille de calcaire (Cleyet-Merle, 1991) provenant du même niveau à sagaie Ccbase et stylistiquement très proche du mammouth gravé sur ivoire de La Madeleine découvert par Lartet et Christy et attribué au Magdalénien récent... A des fins vérificatrices, il fut décidé de procéder à une datation directe en SMA sur un échantillon de la deuxième sagaie à cannelure trouvée dans la couche de Magdalénien moyen des Jamblancs (fig. 3). Les résultats furent (sans appel) : Jamblancs : $17650 \pm 200$ BP (Gif A 97147).

L'âge obtenu, de plusieurs millénaires antérieur à celui des esquilles osseuses précédemment datées (13790 I
120 et $13900 \pm 110$ ) se plaçait incontestablement pendant le Magdalénien ancien. Ce nouveau résultat montrait que le niveau de base étudié avait subi des perturbations dans le passé, fait déjà suspecté lors de travaux de terrain ( Cleyet-Merle, 1991).

Parallèlement, fut effectué le repérage, dans les collections du Musée national de Préhistoire, de termes de comparaison périgourdins, sous la forme de longues et fines sagaies à rainure dorsale : elles étaient présentes, au moins à l'état fragmentaire, à Lascaux et Gabillou entre autres.

Ces objets procédant, au moins apparemment, d'un même contexte et d'une même période, leur analyse pouvait se révéler extrêmement intéressante pour renouveler l'approche chronologique toujours délicate des décors pariétaux de sites totalement dépourvus de datation directe pour les raisons précédemment évoquées.

Deux échantillons complémentaires furent donc soumis à une datation radiocarbone en SMA.

Pour Gabillou, la datation a été obtenue directement sur un fragment de longue sagaie à rainure, objet assez bien représenté dans le mobilier de la cavité (fig. 5) : $17180 \pm$ 170 BP (Gif A 95583).

Rappelons que cet objet provient du matériel archéologique recueilli par Jean Gaussen dans le deuxième sondage pratiqué en 1956, en avant du portail fermant la galerie ornée dont l'entrée avait été fortement modifiée par des aménagements modernes. La couche magdalénienne rencontrée à environ $1,20 \mathrm{~m}$ de profondeur est décrite (Gaussen, 1964) comme sableuse, jaune clair, comportant "de nombreux niveaux archéologiques excessivement minces", et marquée par un fort pendage $\left(45^{\circ}\right)$. La fouille avait été interrompue pour raison de sécurité à 3 mètres sans que fut atteint le substrat rocheux. Le mobilier archéologique avait été attribué dans sa globalité (Gaussen, 1964) au Magdalénien III, sur la base de la présence de ces sagaies à rainure longitudinale.

Pour Lascaux, fut retenu non pas la sagaie à rainure (présente sous forme d'un seul élément distal fig. 4) mais un fragment de baguette en bois de renne. Le résultat est le suivant : Lascaux III, $48: 18600 \pm 190 \mathrm{BP}$ (GIF A 95582).

Ce fragment avait été mis au jour au cours des fouilles menées au pied de la Scène du Puits par H. Breuil, S. Blanc et M. Bourgon, en 1949.

A l'époque, un seul niveau d'occupation avait été remarqué. Son contenu archéologique, relativement abondant pour un secteur aussi reculé et une surface exploitée limitée à quelques mètres carrés seulement, présentait

1. Ou présumés tels dans la mesure où les fouilles récentes ont révélé la présence de décors pariétaux indéterminés sur la paroi Est de l'abri Ouest des Jamblancs. 
des spécificités tant par la nature des objets exhumés que par leur nombre. Ainsi, plus d'une cinquantaine de lampes, non façonnées, voisinaient avec de très nombreuses lamelles à dos et un mobilier osseux relativement abondant, alènes, sagaies, dont une exceptionnelle, de $45 \mathrm{~cm}$ de long (fig.1). Des charbons de conifères, des os fragmentés et des bois de cervidés, dont la plupart réduits en poussière, complétaient le fonds recueilli. L'ensemble était scellé dans un sédiment argilo-sableux étonnamment épais (entre 5 à $15 \mathrm{~cm}$ ) si l'on tient compte de la localisation de ce locus aux tréfonds de cette cavité, dans un secteur où, en principe, la sédimentation est quasiment nulle. L'origine de ces sédiments est à rechercher dans la désagrégation du témoin de remplissage conservé en encorbellement au-dessus de la zone fouillée. On ne peut donc exclure dans ce contexte le téléscopage de vestiges d'époques différentes.

Au cours des prochains mois, la datation directe de la sagaie provenant de ce complexe archéologique permettra de préciser la chronologie du remplissage découvert dans le secteur du Puits.

En conclusion, ces premières mesures, bien que s'échelonnant sur une quinzaine de siècles, et d'autant plus proches qu'elles ont été obtenues sur un type d'objets spécifiques, confirment a priori l'ambiance de très ancien Magdalénien voire de Solutréen récent de la fréquentation des différents gisements concernés.

A Lascaux, elles reculent de 1 à 2 millénaires la période d'occupation du site établie à la suite de mesures effectuées sur charbons de bois récoltés au cours de différents travaux:

- le Puits, fouilles Breuil $1949: 15516 \pm 900 \mathrm{BP}$

- le Puits, fouilles Glory $1959: 16000 \pm 500 \mathrm{BP}$

- le Passage, fouilles Glory $1957: 17190 \pm 140$ BP.

Dans l'état actuel, ces résultats, partiels, basés sur des objets contextuels de l'art pariétal confortent, du moins dans la zone géographique limitée du Périgord, les propositions chronologiques un moment envisagées par Leroi-Gourhan (Leroi-Gourhan, 1965) et confirment leur cohérence avec les autres données fiables - notamment celle fournie par le bloc gravé de Solvieux (Gaussen et Sackett, 1984) - disponibles dans le secteur.

\section{BIBLIOGRAPHIE}

AUJOULAT N. (Sous presse). La répartition des grottes ornées en Périgord noir.

CLEYET-MERLE J.-J., 1989. Nouvelles données sur le Magdalénien de la vallée de la Couze. Paléo, 1. p. 107116.

CLEYET-MERLE J.-J., 1991. Campagne de fouille aux Jamblancs. Rapport d'activité et bilan du programme triennal.

CLEYET-MERLE J.-J., 1992. Le Magdalénien dans la vallée de la Couze et ses origines d'après les fouilles des Jamblancs. In : Peuplement magdalénien. Colloque de Chancelade 1988. Ed. C.T.H.S., Paris. p. 223-234.

CLOTTES J. et al. 1992. La grotte Cosquer datée. Bulletin de la Société préhistorique Française, T. $89, \mathrm{n}^{\circ}$ 8, p. 230-237.

CLOTTES J. et al. 1995. Les peintures paléolithiques de la grotte Chauvet-Pont d'Arc, à Vallon Pont d'Arc (Ardèche, France) : datations directes et indirectes par la méthode du radiocarbone. Comptes-rendus de I'Académie des Sciences de Paris 320 (lia), p. 1133-40.

GAUSSEN J., 1964. La grotte ornée de Gabillou. Imprimerie Delmas, Bordeaux. 68 p., 69 pl.

GAUSSEN J., SACKETT J., 1984. La Pierre gravée de Solvieux. L'Anthropologie, Tome 88, $n^{\circ} 4$. p. 655-660.

LEROI-GOURHAN A., 1965. Préhistoire de l'art occidental. Ed. Mazenod, Paris 1ère édition. 502 p. 804 fig.

LEROI-GOURHAN Arl., ALLAIN J. et al., 1979. Lascaux inconnu. XIle suppll. GALLIA Préhistoire. Ed. CNRS, Paris. 381 p., 387 fig. 27 pl.

PEYRONY D., 1912. Nouvelles fouilles aux ChampsBlancs ou Jean-Blancs. A.F.A.S., Nîmes. p. 522-528.

PEYRONY D., PEYRONY E., 1934. La station préhistorique des Jean-Blancs (Dordogne). B.S.H.A.P. p. 179202.

VALLADAS H. et al., 1992. Direct radiocarbon dates for prehistoric paintings at the Altamira, El castillo and Niaux caves. Nature, 357, p. 68-70. 


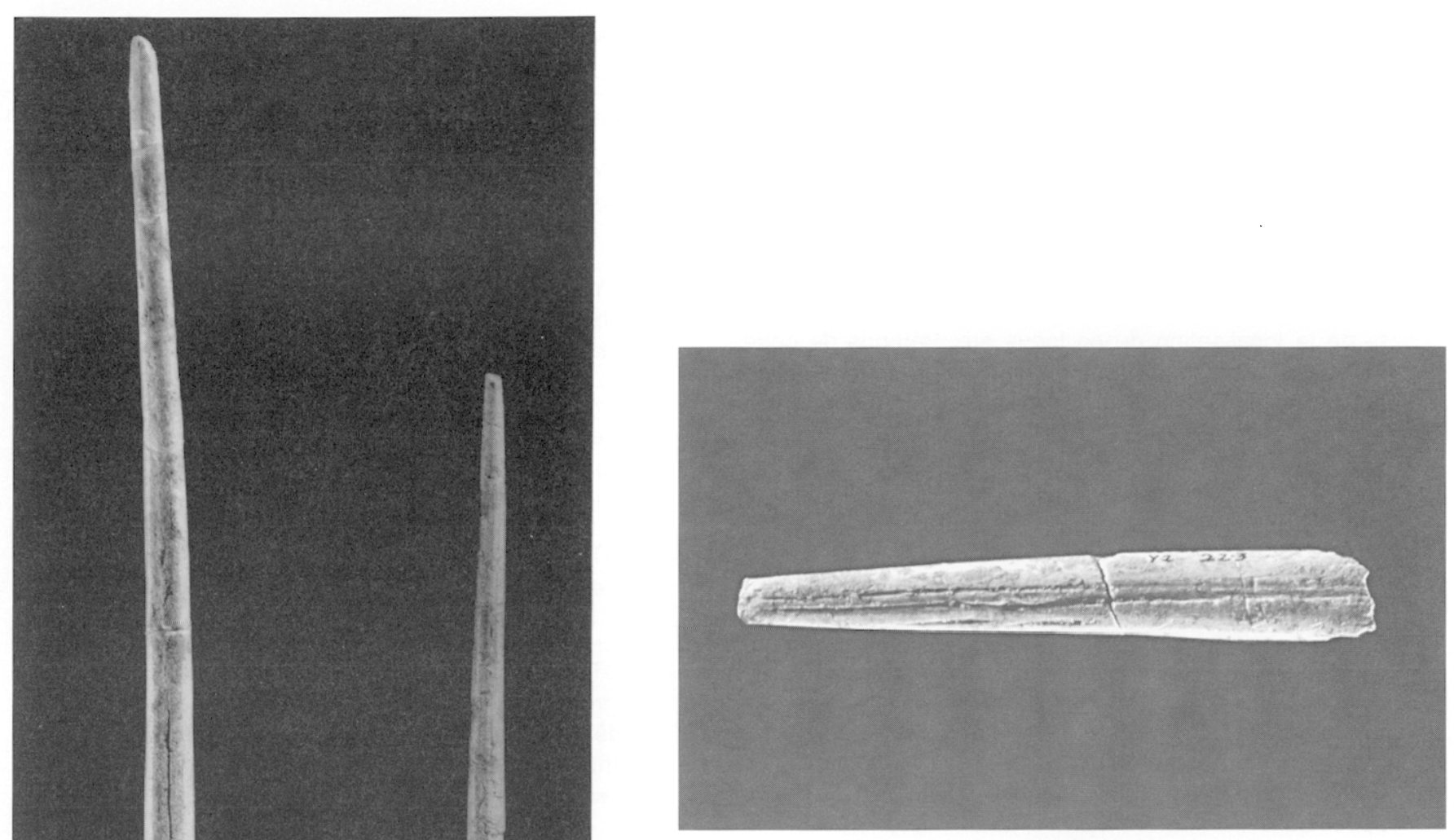

Fig. 3. Jamblancs. Grandeur nature.

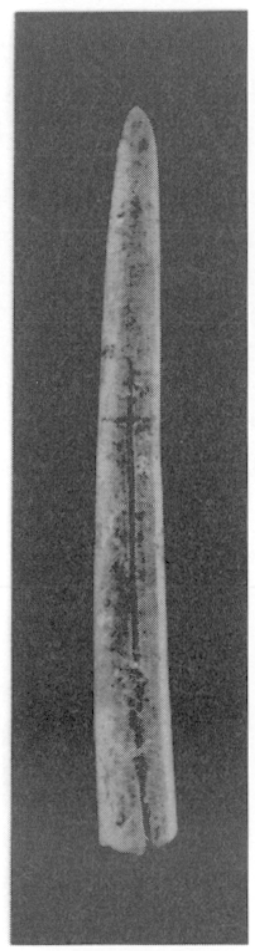

Fig. 1. Lascaux.

1/2 grandeur nature.
Fig. 2. Jamblancs. $1 / 2$ grandeur nature.

Fig. 4. Lascaux. Grandeur nature. 


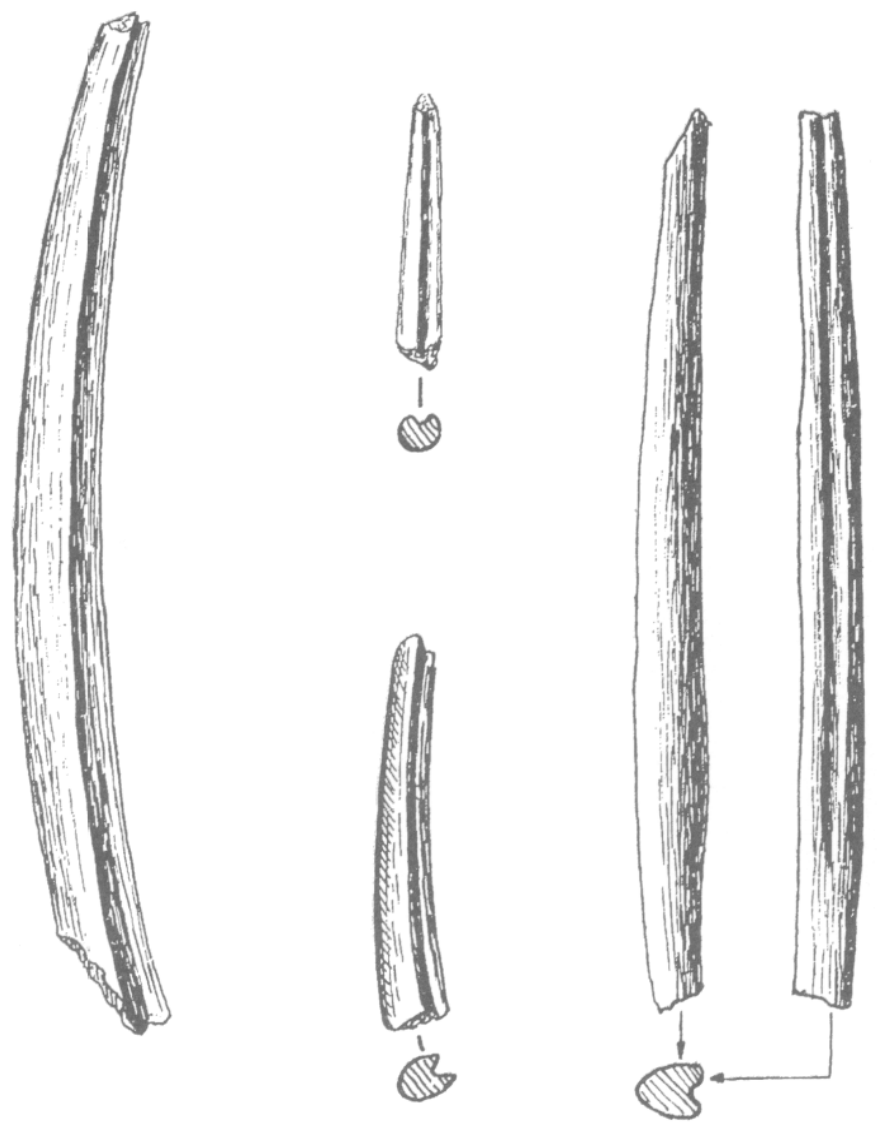

Fig. 5. Gabillou (d'après Gaussen, 1964). 